\title{
Studies of Bending Effects of Microvilli of Leukocyte on Rolling Adhesion
}

\author{
Tai-Hsien $\mathrm{Wu}$ and Dewei Qi* \\ Western Michigan University, Department of Chemical and Paper Engineering, Kalamazoo, USA
}

\begin{abstract}
It has been widely acknowledged that further understanding about the cell adhesion (e.g., leukocyte rolling adhesion) can help us gain more knowledge about the causes of relevant diseases and design more effective treatments and diagnoses. Although recent simulation studies considered the deformability of the leukocytes, most of them, however, did not consider the bending deformation of microvilli. In this paper, an advanced leukocyte model based on an immersed boundary lattice-Boltzmann lattice-spring model (LLM) and an adhesive dynamics (AD) is presented in details. The flexural stiffness of microvilli is introduced into the model for simulations of leukocyte rolling adhesion. This innovative model is applied to investigate the influences of bending deformation of microvilli on the process of leukocyte rolling adhesion and the underlying mechanism at different shear rates. It is demonstrated that the bending deformation of microvilli can be influenced by the flexural stiffness of microvilli and shear rates, resulting in the different rolling velocity of leukocytes, number of receptor-ligand bonds, and bond forces. The findings clearly indicate that the bending of microvilli plays a crucial role in the dynamics of leukocyte adhesion.
\end{abstract}

Submitted June 01, 2017

*Correspondence: dewei.qi@wmich.edu.

Address reprint requests to Dewei Qi, 1903 W. Michigan Ave., Kalamazoo, MI 49009. Tel.: 269-276-3523; Fax: 269-276-3501.

\section{INTRODUCTION}

Cell adhesion plays important roles in various in-vivo and in-vitro binding applications. For example, chimeric antigen receptor (CAR) T-cells rely on binding to cancer cells, under various shear rates, in order to slow or halt potential cancer growth $(1,2)$. A more general example is leukocytes rolling along the inflammation-activated vascular endothelium in blood flow, which is a part of the human immune response. This rolling process is mediated by selectin-ligand bonds, such as P-selectin-P-selectin glycoprotein ligand-1 (PSGL-1) bonds. Many experimental and numerical studies have confirmed that the dynamics of adhesion are mainly mediated by the physical chemistry of adhesion molecules, such as the association and dissociation rates of adhesion bonds $(3,4)$, selectin/ligand density, and the local circulatory environment including flow shear rate $(3,5)$.

In the earliest attempts of simulation, leukocytes were modeled by rigid spheres. However, cellular material properties such as cell deformation play a crucial role in the dynamics of cell adhesion. Khismatullin and Truskey $(6,7)$ first used a compound viscoelastic drop to model neutrophils and monocytes to investigate the effects of cell deformability in a parallel-plate flow chamber. Almost at the same time, Jadhav et al. (8) used a neo-Hookean membrane as a leukocyte, coupled with the immersed boundary method (IBM), to investigate the influence of cell deformability on leukocyte rolling adhesion. Their results indicated that cells with deformations roll much slower and are relatively more stable than those without deformation in shear flow, facts consistent with the experimental findings $(9,10)$. Since then, numerous simulation studies continue to address the process of cell deformation during cellular adhesion (11-16). 
Another critical cellular property is microvillus deformation. Microvilli are fingerlike projections on the surface of leukocytes. Since most adhesion molecules (e.g., PSGL-1) are located at the tips of microvilli, microvillus deformation also affects cellular adhesion. Hence, several simulation studies were performed to examine the influence of the extensional deformation of microvilli on the dynamics of cell adhesion. Khismatullin and Truskey (6) combined the microvillus spring with the receptor-ligand bond spring to express the total extensional deformation of microvilli and adhesive bonds. Later, Caputo and Hammer (17) improved Hammer and Apte's pioneering adhesive dynamics (AD) (3) by using an elastic spring and a viscous model to approximate the microvilli elastic and viscous responses to a small force and a large force, respectively. This idea was first proposed by Shao et al. (18). Pawar et al. (19) further improved Caputo and Hammer's model by replacing the hard spherical body with a deformable neo-Hookean membrane. Recently, Pospieszalska et al. combined the existing model for microvillus tether formation with a Kelvin-Voigt viscoelastic model for microvillus extension $(20,21)$. However, these studies were limited to microvillus extensional deformation.

In fact, when a force tangential to the cell surface is exerted on a leukocyte microvillus, it will be bent. This phenomenon was observed in experiments (22). Similar to the extensional deformation of microvilli, the bending of microvilli due to their flexibility can potentially influence the binding between selectins and ligands. Munn et al. (23) suggested that when microvilli are flattened along the cell surface, the receptors at the bases of microvilli (instead of at the tips of microvilli) may obtain additional adhesion with ligands on the endothelial cells or on the ligand-coated substrate. While Pospieszalska et al. first simulated the pivoting of microvilli around their bases, they did not address the flexural stiffness of microvilli $(20,21)$. Based on the importance of flexibility in microvilli, Yao and Shao $(24,25)$ experimentally measured the flexural stiffness of the microvilli on lymphocytes and neutrophils under small deformation of the microvilli. They reported that the values of the flexural stiffness of lymphocyte and neutrophil microvilli are $4 p N / \mu m$ and $7 p N / \mu m$, respectively $(24,25)$. Simon et al. (26) conducted another experiment to measure the flexural stiffness of neutrophil microvilli under a large deformation $(0.5-1 \mu m)$ and found that the flexural stiffness is $5 p N / \mu m$.

To take account of the bending effects of microvilli, employing the measured data of microvillus flexural stiffness identified by Yao and Shao $(24,25)$, Wu and Qi (27) recently used the immersed boundary lattice-Boltzmann lattice-spring model (LLM) combined with adhesive dynamics simulations (3) to study the roles of flexural stiffness on cell rolling and adhesion at a shear rate of $\gamma=50 \mathrm{~s}^{-1}$. The results showed that the flexural stiffness has a profound effect on rolling velocity and adhesion bonding force. In their studies, they decomposed each individual adhesion bonding force into two local coordinate-based components: parallel (for extension) and perpendicular (for bending). Their findings revealed that the increasing total local coordinate-based adhesion bonding force is mainly due to an increase in the perpendicular component. The flexibility of microvilli aids not only in expediting contact frequency with the substrate, but also in flattening of microvilli once the contact is established. As a result, the flexibility facilitates the formation of adhesive bonds, confirming Munn et al's speculation (23).

This paper advances the study of microvillus flexibility in leukocyte rolling adhesion under different shear rates. In simulations, the statistical ensemble average results of individual microvilli are computed and used to understand the dynamic adhesive behavior, which is difficult to observe in experiments because of limited spatial and temporal resolution currently available. The results suggest that the flexural stiffness of microvilli plays a key role in altering the rolling velocity of leukocytes at different shear rates. As the flexural stiffness of microvilli increases or its flexibility decreases, the leukocytes roll faster or detach from the selectin-coated substrate more easily. This phenomenon is apparent even at a higher shear rate.

The same simulation method developed by Wu and Qi (27) is adopted in the present work. This method is comprised of five numerical models: (1) lattice Boltzmann method (LBM) for the Navier-Stokes flow (28-37); (2) coarse-grained cell model (CGCM) for the viscoelastic leukocyte membrane (13, 38-41); (3) lattice spring model (LSM) for the flexible microvilli (42); (4) immersed boundary method (IBM) for the fluid-cell interactions (43); (5) adhesive dynamics (AD) for stochastic binding between P-selectin and PSGL-1 (3). The method with each model is presented in details in the next section, and the contents were neither reported nor published before.

\section{COMPUTATION METHOD}

A lattice-Boltzmann lattice-spring method $(\operatorname{LLM})(27,42,44-46)$ combined with the AD is employed. In this method, the LBM is used as a fluid solver to simulate the Navier-Stoke flows, the CGCM (38) is utilized to simulate motion of leukocytes, and the LSM (27) is exploited to mimic the deformation of microvilli while the fluid-solid

Biophysical Journal 00(00) 1-22 
interactions are treated by IBM. Further, the AD is employed to obtain the adhesive bonding forces between leukocytes and selectin-coated substrates.

\section{Lattice Boltzmann method}

The motion of Newtonian fluid is governed by the continuity and Navier-Stokes equations as

$$
\begin{gathered}
\nabla \cdot \mathbf{u}=0 \\
\rho_{f}\left[\frac{\partial \mathbf{u}}{\partial t}+(\mathbf{u} \cdot \nabla \mathbf{u})\right]=-\nabla p+\mu \nabla^{2} \mathbf{u}+\mathscr{F}
\end{gathered}
$$

where $\mathbf{u}$ is the fluid velocity; $\rho_{f}$ the fluid density; $p$ the pressure; $\mu$ the fluid dynamic viscosity; and $\mathscr{F}$ the force source term. In this study, the partial differential equations (Eqs. 1 and 2) are not solved directly. Instead, the LBM is used to obtain fluid flow behavior. Previous work has demonstrated that the solution of the LBM is equivalent to that of the Navier-Stokes equations when the Mach number is smaller than 0.3 (30). Importantly, the kinetic nature of the LBM makes it more suitable for simulating the multi-component flows such as blood (47-51). More features of the LBM can be referred to previous studies (35).

The multiple-relaxation-time (MRT) lattice Boltzmann equation (52) in a D3Q19 lattice model with a forcing term is used and expressed as

$$
\boldsymbol{f}\left(\mathbf{x}+\mathbf{e}_{i} \delta t, t+\delta t\right)-\boldsymbol{f}(\mathbf{x}, t)=-\mathbf{M}^{-1} \mathbf{S}\left(\mathbf{m}(\mathbf{x}, t)-\mathbf{m}^{e q}(\mathbf{x}, t)\right)+\delta t \mathbf{M}^{-1} \widehat{\boldsymbol{F}},
$$

where $\boldsymbol{f}(\mathbf{x}, t)$ and $\mathbf{m}(\mathbf{x}, t)$ are the fluid distribution functions at position $\mathbf{x}$ and time $t$ in the velocity and moment spaces, respectively; $\mathbf{m}^{e q}$ denotes the equilibrium distribution function in moment space; $\delta t$ represents the time interval; $\mathbf{e}_{i}$ is the discrete velocity set where $i \in\{0,1,2, \ldots, 18\}$ are the discrete directions; $\mathbf{S}$ is the diagonal collision matrix; $\mathbf{M}$ is the transformation matrix (given in Ref. (52) Appendix) which transfers the distribution functions from the velocity space into the moment space; and $\widehat{\boldsymbol{F}}$ is the moment of the forcing term in the moment space.

In the D3Q19 model, the discrete velocity set $\mathbf{e}_{i}$ is written as:

$$
\mathbf{e}_{i}= \begin{cases}(0,0,0) & i=0 \\ ( \pm 1,0,0),(0, \pm 1,0),(0,0, \pm 1) & i=1-6 \\ ( \pm 1, \pm 1,0),( \pm 1,0, \pm 1),(0, \pm 1, \pm 1) & i=7-18\end{cases}
$$

and diagonal collision matrix $\mathbf{S}$ is written as:

$$
\mathbf{S}=\left(0, s_{e}, s_{\epsilon}, 0, s_{q}, 0, s_{q}, 0, s_{q}, s_{\nu}, s_{\pi}, s_{\nu}, s_{\pi}, s_{\nu}, s_{\nu}, s_{\nu}, s_{t}, s_{t}, s_{t}\right)
$$

while shear and bulk viscosities are given by

$$
\begin{aligned}
& \nu=\frac{1}{3}\left(\frac{1}{s_{\nu}}-\frac{1}{2}\right) \delta t, \\
& \zeta=\frac{2}{9}\left(\frac{1}{s_{e}}-\frac{1}{2}\right) \delta t,
\end{aligned}
$$

where $s_{\nu}$ is related to the shear viscosity. As suggested by Pan et al. (53), the other relaxation rates are set as $s_{e}=s_{\epsilon}=s_{\pi}=s_{\nu}$ and $s_{t}=s_{q}=8 \cdot \frac{2-s_{\nu}}{8-s_{\nu}}$.

The distribution functions in the moment space $\mathbf{m}$ and the corresponding equilibria $\mathbf{m}^{e q}$ are written by

$$
\begin{gathered}
\mathbf{m}=\left(\rho_{f}, e_{f}, \epsilon_{f}, j_{x}, q_{x}, j_{y}, q_{y}, j_{z}, q_{z}, 3 p_{x x}, 3 \pi_{x x}, p_{w w}, \pi_{w w}, p_{x y}, p_{y z}, p_{z x}, t_{x}, t_{y}, t_{z}\right)^{T}, \\
\mathbf{m}^{e q}=\rho_{f}\left(1,-11+19 u^{2}, \alpha_{f}+\beta_{f} u^{2}, u_{x},-\frac{2}{3} u_{x}, u_{y},-\frac{2}{3} u_{y}, u_{z},-\frac{2}{3} u_{z}, 3 u_{x}^{2}-u^{2}, \frac{\gamma_{f} p_{x x}^{e q}}{\rho}, u_{y}^{2}-u_{z}^{2}, \frac{\gamma_{f} p_{w w}^{e q}}{\rho},\right. \\
\left.u_{x} u_{y}, u_{y} u_{z}, u_{z} u_{x}, 0,0,0\right)^{T}
\end{gathered}
$$

where $e_{f}$ and $\epsilon_{f}$ represent energy and energy squared; $j_{x, y, z}$ are components of the momentum; $q_{x, y, z}$ are components of the heat flux; $p_{x y, y z, z x}$ are the symmetric and traceless strain-rate tensor; $\pi_{x x, w w}$ are the fourth order moments and $t_{x, y, z}$ are the third order moments $(52) ; u^{2}=u_{x}^{2}+u_{y}^{2}+u_{z}^{2}$ denotes the fluid velocity squared; and the three parameters $\alpha_{f}=3, \beta_{f}=\frac{-11}{2}$, and $\gamma_{f}=\frac{-1}{2}$ are chosen. 
Furthermore, according to the work by Guo et al. $(54,55)$, the moment of the forcing term in the moment space $\widehat{\boldsymbol{F}}$ can be written as,

$$
\widehat{\boldsymbol{F}}=\left(\mathbf{I}-\frac{1}{2} \mathbf{S}\right) \mathbf{M} \overline{\boldsymbol{F}}
$$

where $\mathbf{I}$ is the unity matrix; $\overline{\boldsymbol{F}}=\left(\bar{F}_{0}, \bar{F}_{1}, \ldots, \bar{F}_{18}\right)^{T}$ and

$$
\bar{F}_{i}=\omega_{i}\left(\frac{\mathbf{e}_{i} \cdot \boldsymbol{F}}{c_{s}^{2}}+\frac{\mathbf{u} \boldsymbol{F}:\left(\mathbf{e}_{i} \mathbf{e}_{i}-c_{s}^{2} \mathbf{I}\right)}{c_{s}^{4}}\right),
$$

where $\boldsymbol{F}$ is the body force; $c_{s}=\sqrt{\frac{1}{3}}$ is the sound speed; $\omega_{i}$ is the weight associated with the lattice model and defined by

The fluid velocity $\mathbf{u}$ is given by

$$
\omega_{i}= \begin{cases}\frac{1}{3} & i=0 \\ \frac{1}{18} & i=1-6 \\ \frac{1}{36} & i=7-18\end{cases}
$$

$$
\rho_{f} \mathbf{u}=\sum_{i} f_{i} \mathbf{e}_{i}+\frac{1}{2} \delta t \boldsymbol{F} .
$$

All parameters used in the LBM are given in Table 1 and Table 2.

\section{Immersed boundary method}

In the LBM, the fluid particles are in regular Eulerian grids, whereas the solid particles are in Lagrangian grids. Therefore, a solid grid may not coincide with its adjacent fluid grids. The IBM is used to achieve non-slip boundary condition in the fluid-solid interfaces. For the purpose, a discrete Dirac delta function $\delta^{D}$ is taken to interpolate the fluid velocity at the position of a solid boundary grid from its surrounding fluid grids. The discrete Dirac delta function (43) is written by

$$
\delta^{D}(\Delta \mathbf{r})= \begin{cases}\frac{1}{64 h^{3}}\left(1+\cos \frac{\pi \Delta x}{2 h}\right)\left(1+\cos \frac{\pi \Delta y}{2 h}\right)\left(1+\cos \frac{\pi \Delta z}{2 h}\right) & |\Delta \mathbf{r}| \leq 2 h \\ 0 & |\Delta \mathbf{r}|>2 h\end{cases}
$$

where $h$ is the fluid grid spacing and $\Delta \mathbf{r}=(\Delta x, \Delta y, \Delta z)$ is the distance between the positions of the solid boundary grid and its surrounding fluid grids.

To present the IBM, the solid boundary domain $\Gamma$ and fluid boundary domain $\Pi$ are defined. The solid boundary domain $\Gamma$ is constituted by all the solid grids on the surface, and the fluid boundary domain $\Pi$ is defined as a spherical volume of a radius of $2 h$ centered at a solid boundary grid position $\mathbf{r}^{b}$. The un-forced fluid velocity $\mathbf{u}^{*}$ at $\mathbf{r}^{b}$ is represented by

$$
\mathbf{u}^{*}\left(\mathbf{r}^{b}, t\right)=\int_{\Pi} \mathbf{u}^{*}(\mathbf{r}, t) \delta^{D}\left(\mathbf{r}-\mathbf{r}^{b}\right) d \mathbf{r},
$$

where $\mathbf{r}$ is a variable and goes all the positions of the fluid grids in the fluid domain $\Pi$ during the integration. Due to the no-slip boundary condition, the forced fluid velocity $\mathbf{u}\left(\mathbf{r}^{b}, t\right)$ should be equal to the solid velocity $\mathbf{U}^{b}\left(\mathbf{r}^{b}, t\right)$, as follows:

$$
\mathbf{u}\left(\mathbf{r}^{b}, t\right)=\mathbf{u}^{*}\left(\mathbf{r}^{b}, t\right)+\delta t \frac{\mathcal{F}\left(\mathbf{r}^{b}, t\right)}{\rho_{f}}=\mathbf{U}^{b}\left(\mathbf{r}^{b}, t\right)
$$

where $\mathcal{F}$ is the fluid-solid interaction force exerted on the fluid. Therefore, the interaction force $\mathcal{F}$ exerted on the fluid at the solid boundary position can be expressed by

$$
\mathcal{F}\left(\mathbf{r}^{b}, t\right)=\frac{\rho_{f}\left(\mathbf{U}^{b}\left(\mathbf{r}^{b}, t\right)-\mathbf{u}^{*}\left(\mathbf{r}^{b}, t\right)\right)}{\delta t} .
$$

Thus, the interaction force acting on the solid grids by the surrounding fluid is given by

$$
\boldsymbol{F}^{H}\left(\mathbf{r}^{b}, t\right)=-\mathcal{F}\left(\mathbf{r}^{b}, t\right)
$$

The discrete Dirac delta function is utilized again to distribute the interaction force $\mathcal{F}\left(\mathbf{r}^{b}, t\right)$ to the surrounding fluid grids by

$$
\boldsymbol{F}(\mathbf{r}, t)=\int_{\Gamma} \mathcal{F}\left(\mathbf{r}^{b}, t\right) \delta^{D}\left(\mathbf{r}-\mathbf{r}^{b}\right) d \mathbf{r}^{b}
$$

where $\boldsymbol{F}(\mathbf{r}, t)$ is the distributed body force and can be used in Eq. 11 .

Biophysical Journal 00(00) 1-22 


\section{Coarse-grained cell model}

A leukocyte can be simulated by a coarse-grained cell model (CGCM), presented by Fedosov et al. (38), in which the leukocyte is assumed to be a spherical membrane. The membrane is considered as a two-dimensional triangulated network. An open source MATLAB mesh generator presented by Persson et al. (56) was used in this study for creating meshes.

In the triangulated network, $N_{p}$ denotes the number of solid grids, $N_{b}$ the number of edges, and $N_{e}$ the number of triangles. Four types of potential energies are calculated between solid grids. The total potential energy of the membrane can be written as

$$
U^{\text {total }}=U^{\text {in-plane }}+U^{\text {area }}+U^{\text {volume }}+U^{\text {bending }} .
$$

The in-plane potential energy term $U^{\text {in-plane }}$ has several formulas constituted by two-body and three-body energies. In this study, an edge is considered as a non-linear spring, whose energy is constituted by the combination of the finitely extensible nonlinear elastic (FENE) and power law (POW) potential energies, as follows:

$$
U^{i n-p l a n e}=\sum_{j}^{N_{b}}-\frac{1}{2} k_{F} R_{0}^{2} \ln \left(1-\left(\frac{r}{R_{0}}\right)^{2}\right)+\frac{k_{P}}{(m-1) r^{m-1}} \quad m>0 \text { and } m \neq 1,
$$

where $k_{F}$ is a constant coefficient of the FENE potential; $R_{0}$ and $r$ are the maximum and instant distances between two solid grids, respectively; $k_{P}$ is the coefficient of the POW potential energy; and, $m$ is the exponent of the power law. Apparently, $U^{\text {in-plane }}$ is only a two-body energy of a non-linear spring.

The area conservation and volume conservation energies, $U^{\text {area }}$ and $U^{\text {volume }}$, are the three-body energies which exist in a triangle constituted by three neighboring solid grids. The area and volume conservation energies are defined as follows:

$$
\begin{gathered}
U^{\text {area }}=\frac{k_{A}\left(A-A_{0}^{\text {tot }}\right)^{2}}{2 A_{0}^{\text {tot }}}+\sum_{j}^{N_{e}} \frac{k_{D}\left(A_{j}-A_{j}^{0}\right)^{2}}{2 A_{j}^{0}}, \\
U^{\text {volume }}=\frac{k_{V}\left(V-V_{0}^{\text {tot }}\right)^{2}}{2 V_{0}^{\text {tot }}},
\end{gathered}
$$

where $k_{A}, k_{D}$, and $k_{V}$ are the global area, local area, and volume constraint constants, respectively. The term $A$ and $V$ represent the instantaneous entire area and volume, respectively, while $A_{0}^{\text {tot }}$ and $V_{0}^{\text {tot }}$ are the initial total area and volume; $A_{j}^{0}$ and $A_{j}$ are the initial and instantaneous local area of the $j$ th triangle.

Furthermore, the bending energy $U^{\text {bending }}$ exists between two adjacent triangles (four adjacent solid grids) and is given by the following equation:

$$
U^{\text {bending }}=\sum_{j}^{N_{b}} k_{B}\left[1-\cos \left(\theta_{j}-\theta_{0}\right)\right],
$$

where $k_{B}$ is the bending coefficient; and $\theta_{j}$ and $\theta_{0}$ are the instantaneous and initial angles, respectively, between two adjacent triagnels which have the common edge $j$.

The total elastic force $\boldsymbol{F}_{i}^{E}$ exerted on the $i$ th grid in the CGCM is computed by

$$
\boldsymbol{F}_{i}^{E}=-\nabla_{i}\left(U^{\text {in-plane }}+U^{\text {area }}+U^{\text {volume }}+U^{\text {bending }}\right) .
$$

The gradient is calculated analytically, and the answers are used in the code.

The CGCM $(13,38-41)$ also addresses the membrane viscosity by adding the dissipative $\boldsymbol{F}_{i j}^{D}$ for each spring (edge) as

$$
\boldsymbol{F}_{i j}^{D}=-\eta^{T} \mathbf{v}_{i j}-\eta^{C}\left(\mathbf{v}_{i j} \cdot \mathbf{e}_{i j}\right) \mathbf{e}_{i j},
$$

where $\eta^{T}$ and $\eta^{C}$ are dissipative coefficients, respectively; and $\mathbf{v}_{i j}$ and $\mathbf{e}_{i j}$ are the relative velocity and unit vector, respectively, between the $i$ th and $j$ th grids. The membrane shear viscosity $\eta^{m}$ is related to the dissipative parameters $\left(\eta^{T}\right.$ and $\left.\eta^{C}\right)$, as follows:

$$
\begin{gathered}
\eta^{m}=\sqrt{3} \eta^{T}+\frac{\sqrt{3}}{4} \eta^{C}, \\
\eta^{C}=\frac{\eta^{T}}{3} .
\end{gathered}
$$


A repulsive force is added on leukocytes between the leukocytes and substrate in the Z-direction (perpendicular to the substrate) when the leukocytes are close to the substrate. The repulsive force is borrowed from the gradient of the Lennard-Jones potential $\left(U^{L J}\right)$ with negative sign, as follows:

$$
\boldsymbol{F}^{R}(\delta z)= \begin{cases}-\nabla_{z}\left\{U^{L J}(\delta z)\right\}=-\nabla_{z}\left\{4 \epsilon_{R}\left[\left(\frac{\sigma_{R}}{\delta z}\right)^{12}-\left(\frac{\sigma_{R}}{\delta z}\right)^{6}\right]\right\} & \delta z \leq R^{C} \\ 0 & \delta z>R^{C}\end{cases}
$$

where $\delta z$ is the distance above the substrate; $R^{C}$ is the cut-off radius; $\epsilon_{R}$ is the well depth of the potential; and $\sigma_{R}$ is the distance between the substrate surface and the position where $U^{L J}=0$. Therefore, the total force exerted on the $i$ th solid grid is given by

$$
\boldsymbol{F}_{i}^{T}=\boldsymbol{F}_{i}^{E}+\sum_{j} \boldsymbol{F}_{i j}^{D}+\boldsymbol{F}_{i}^{H}+\boldsymbol{F}_{i}^{R},
$$

where $\boldsymbol{F}^{T}, \boldsymbol{F}^{E}, \boldsymbol{F}^{D}, \boldsymbol{F}^{H}$, and $\boldsymbol{F}^{R}$ denote the total, elastic, dissipative, hydrodynamic, and repulsive forces, respectively; and $j$ denotes the grid nearby the $i$ th solid grid. Once the total force of a solid grid is obtained, the leap-frog algorithm is used to obtain the position and velocity of each solid grid during the simulation run.

Next, six parameters $\left(k_{F}, k_{P}, k_{A}, k_{D}, k_{V}\right.$, and $\left.k_{B}\right)$ must be determined in the CGCM. First, the potential coefficient of POW $k_{P}$ can be described in terms of $k_{F}$ since the attractive term should be equal to the repulsive term at the equilibrium point in $U^{i n-p l a n e}$. By setting the exponent of the power law $m=6$ and $R_{0}=1.75 r$, the potential coefficient of power law $k_{P}$ can be written by

$$
k_{P} \cong 1.4848 k_{F} r^{m+1}=1.4848 k_{F} r^{7}
$$

Secondly, according to Fedosov et al. $(38,39)$, the shear stress $\mu_{0}$ based on the $U^{i n-p l a n e}$ is given by

$$
\mu_{0}=\frac{\sqrt{3}}{4}\left\{\frac{2 k_{F}\left(\frac{r}{R_{0}}\right)^{2}}{\left[1-\left(\frac{r}{R_{0}}\right)^{2}\right]^{2}}+\frac{k_{P}(m+1)}{r^{m+1}}\right\}
$$

and the compression modulus $\kappa$ of cell is given by

$$
\kappa=2 \mu_{0}+k_{A}+k_{D}
$$

The linear Young's modulus $Y$ and Poisson's ratio $\nu^{p}$ of the cell are expressed by

$$
\begin{aligned}
Y & =\frac{4 \kappa \mu_{0}}{\kappa+\mu_{0}}, \\
\nu^{p} & =\frac{\kappa-\mu_{0}}{\kappa+\mu_{0}} .
\end{aligned}
$$

To achieve incompressibility and to make $k_{A}+k_{D}>>\mu_{0}$, Fedosov et al. showed that a nearly incompressible membrane is achieved when $k_{A}+k_{D}=500 \mu_{0}$. The assumption is followed. In addition, the bending coefficient $k_{B}$ in the CGCM is given by

$$
k_{B}=\frac{2}{\sqrt{3}} k_{C}
$$

where $k_{C}$ is the bending rigidity. All parameters used in the CGCM are collected in Table 1 and Table 2.

\section{Lattice spring model}

Wu and Qi (27) applied the LSM (42) to mimic the deformation of the leukocyte microvilli. As shown in Fig. 1, a microvillus is modeled by a spring (vector $\mathbf{r}_{j i}$ ) with its end fixed at the base grid $i$ on the cell surface and with the other end $j$ as the microvillus tip. Note that the base grids in the LSM are also the solid grids in the CGCM. There are two types of deformation of microvilli. First, the tip grid $j$ of the spring can be extended or compressed with respect to the base grid $i$. The potential energy between grids $i$ and $j$ responsible for the spring extension or compression $U_{j i}^{s}$ is given by

$$
U_{j i}^{s}=\frac{1}{2} k_{s}^{m v}\left(r_{j i}-r_{0 j i}\right)^{2},
$$

Biophysical Journal 00(00) 1-22 


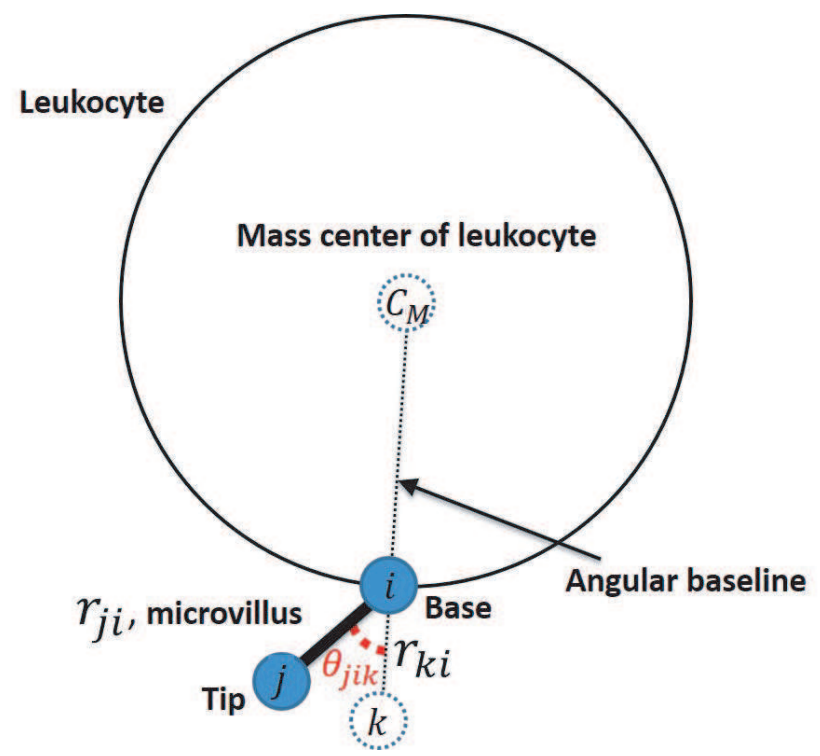

Figure 1: A microvillus is modeled by a spring with its end fixed at the base node $i$ on the cell surface. The tip node $j$ can be extended or compressed with respect to the base node $i$. In addition, the vector $\mathbf{r}_{j i}$ can be bent with respect to vector $\mathbf{r}_{k i}$ to have a bending angle $\theta_{k i j}$, where the node $k$ is always located on the extension portion of the line connecting the mass center $C_{M}$ to the base node $i$.

where $r_{j i}$ and $r_{0 j i}$ are the instantaneous and initial microvillus lengths, respectively; and $k_{s}^{m v}$ is the extensional spring constant. Note that each microvillus has the same initial length (i.e., $r_{0 j i}=L_{m v}$, where $L_{m v}=0.35 \mu m$ is the average experimental value of microvillus length).

Second, vector $\mathbf{r}_{j i}$ can be bent with respect to vector $\mathbf{r}_{k i}$ to have a bending angle $\theta_{k i j}$, where $k$ is always located on the extension portion of the line connecting the mass center $C_{M}$ to the base grid $i$. The line is called the angular baseline (the dashed line in Fig. 1) and is used for the measurement of the bending angle only. In fact, $\mathbf{r}_{k i}$ is a virtual bond, and grid $k$ does not participate in simulation. The energy due to the bent bond among three particles $U_{k i j}^{a}$ is written by:

$$
U_{k i j}^{a}=\frac{1}{2} k_{a}^{m v}\left(\theta_{k i j}-\theta_{0 k i j}\right)^{2}
$$

where $k_{a}^{m v}$ is the angular spring constant, and $\theta_{0 k i j}$ represents the equilibrium angle, as shown in Fig. 1 . The elastic forces due to the extensional and bending deformation are calculated from the negative gradient of the sum of $U_{j i}^{a}$ and $U_{k i j}^{s}$ with respect to solid grids $i$ and $j$.

Base on previous experimental measurements and numerical studies $(9,24-26)$, the extensional stiffness and flexural stiffness of the microvilli are $152-1340 p N / \mu m$ and $4-7 p N / \mu m$, respectively. The extensional stiffness $K^{E}$ is directly equal to the spring constant $k_{s}^{m v}$, while the flexural stiffness $K$ is defined by a ratio of the force on the microvillus to the corresponding deflection, and it is related to the angular spring constant by $K=k_{a}^{m v} / L_{m v}^{2}(27)$. All parameters used in the LSM are given in Table 1 and Table 2.

\section{Adhesive dynamics}

The AD was first presented in a simulation of leukocyte adhesion to endothelial cells by Hammer and Apte (3). This method uses the stochastic Monte Carlo method coupled with kinetics models to simulate the formation and rupture of the receptor-ligand bond.

The kinetics model used in this study is the Dembo model (57), where the forward and reverse rates for the receptor-ligand bond are written as follows:

$$
k_{f}=k_{f}^{0} \exp \left[-\frac{\sigma_{t s}\left(l-l_{0}\right)^{2}}{2 K_{B} T}\right],
$$




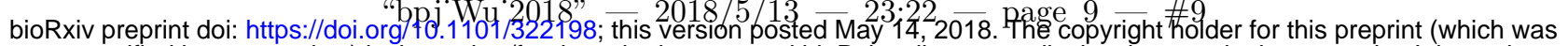

not certified by peer review) is the author/funder, who has granted bioRxiv a license to display the preprint in perpetuity. It is made available under aCC-BY 4.0 International license.

Table 2: The simulation dimensionless parameters used in this study.

\begin{tabular}{|c|c|c|}
\hline Parameter & Definition & Value \\
\hline \multicolumn{3}{|l|}{$\overline{\mathrm{LBM}}$} \\
\hline$\rho_{f}$ & Fluid density & 1 \\
\hline$\nu_{f}$ & Fluid kinematic viscosity & 0.64 \\
\hline$\gamma$ & Shear rate & $(1.512,3.024,4.536,6.048) \times 10^{-5}$ \\
\hline$\left(N_{x}, N_{y}, N_{z}\right)$ & Simulation box & $(32,48,32)$ \\
\hline$\left(s_{e}, s_{\epsilon}, s_{\pi}, s_{\nu}, s_{t}, s_{q}\right)$ & Relaxation rates & $(0.41,0.41,0.41,0.41,1.67,1.67)$ \\
\hline$\left(\alpha_{f}, \beta_{f}, \gamma_{f}\right)$ & Three free parameters in the MRT & $(3,-5.5,-0.5)$ \\
\hline \multicolumn{3}{|l|}{ CGCM } \\
\hline$N_{p}$ & Number of solid nodes & 714 \\
\hline$\rho_{W B C}$ & Leukocyte density & 1.077 \\
\hline$Y$ & Leukocyte membrane Young's modulus & 0.0454 \\
\hline$\mu_{0}$ & Leukocyte membrane shear stress & 0.0116 \\
\hline$k_{C}$ & Leukocyte membrane bending rigidity & 0.0016 \\
\hline$\left(\eta^{T}, \eta^{C}\right)$ & dissipative parameters & $\left(1.9896 \times 10^{-4}, 6.6321 \times 10^{-5}\right)$ \\
\hline$m$ & Exponent in POW & 6 \\
\hline$k_{F}$ & FENE coefficient & $2.298 \times 10^{-3}$ \\
\hline$k_{A}$ & Global area constant & 0.294 \\
\hline$k_{D}$ & Local area constant & 0.00589 \\
\hline$k_{V}$ & Volume constant & 0.294 \\
\hline$k_{B}$ & Bending coefficient & 0.00185 \\
\hline$R^{C}$ & Cut-off radius of the repulsive potential & 0.055 \\
\hline$\sigma_{R}$ & Well depth of the repulsive potential & 0.05 \\
\hline$\epsilon_{R}$ & Distance where repulsive potential equals zero & $2 \times 10^{-5}$ \\
\hline \multicolumn{3}{|c|}{ 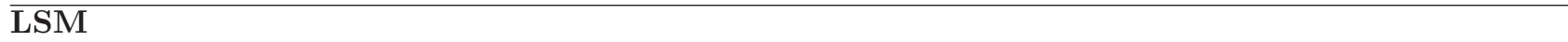 } \\
\hline$N_{m v}$ & Number of microvilli & 714 \\
\hline$L_{m v}$ & Microvillus length & 0.6571 \\
\hline$k_{s}^{m v}$ & Microvillus spring constant & 0.203 \\
\hline$k_{a}^{m v}$ & Microviilus angle constant & $\left(4.58 \times 10^{-5}, 4.58 \times 10^{-4}, 4.58 \times 10^{-3}\right)$ \\
\hline \multicolumn{3}{|r|}{ C } \\
\hline$k_{f}^{0}$ & Unstressed forward rate & $1.51287 \times 10^{-7}$ \\
\hline$k_{r}^{0}$ & Unstressed reverse rate & $1.51287 \times 10^{-7}$ \\
\hline$\sigma_{b}$ & Bond spring constant & 0.151495 \\
\hline$\sigma_{t s}$ & Transition state spring constant & 0.14998 \\
\hline$l_{0}$ & Equilibrium bond length & 0.1877 \\
\hline \multirow[t]{2}{*}{$K_{B} T$} & Thermal energy & $2.2858 \times 10^{-6}$ \\
\hline & P-selectin site density & 43 \\
\hline$\Delta t$ & Time interval for adhesion determination & 1000 \\
\hline$A_{L} n_{L}$ & Number of PSGL-1 accessible to each receptor & 5 \\
\hline
\end{tabular}

\section{RESULTS AND DISCUSSION}

\section{Simulation setup}

In this study, the leukocyte rolling processes in shear flow are simulated at shear rates of $\gamma=100-400 s^{-1}$, as shown in Fig. 2. The simulation box is $32 \times 46 \times 32$, where a fluid grid spacing is $5.326 \times 10^{-7} \mathrm{~m}$, and a simulation time step is $1.512 \times 10^{-7} \mathrm{~s}$. A whole leukocyte consists of 714 solid grids for the cell membrane (and also for the microvilli bases), another 714 solid grids for the microvilli tips, and 714 springs for the microvilli. The average membrane particle spacing is $5.326 \times 10^{-7} \mathrm{~m}$, the same as the fluid grid spacing. Two substrates are placed at the bottom and top of the simulation box in the Z-direction. The bottom substrate is at rest and coated with P-selectin at a density of $150 / \mathrm{\mu m}^{-2}$. A velocity of $\gamma H_{s}$ is imposed on the top substrate, where $H_{s}$ is the distance between the 


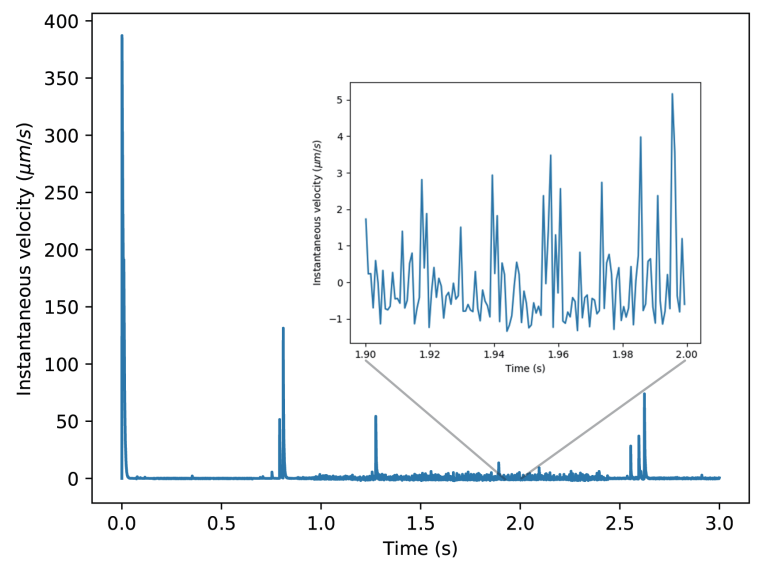

(a) $\gamma=100 s^{-1}$

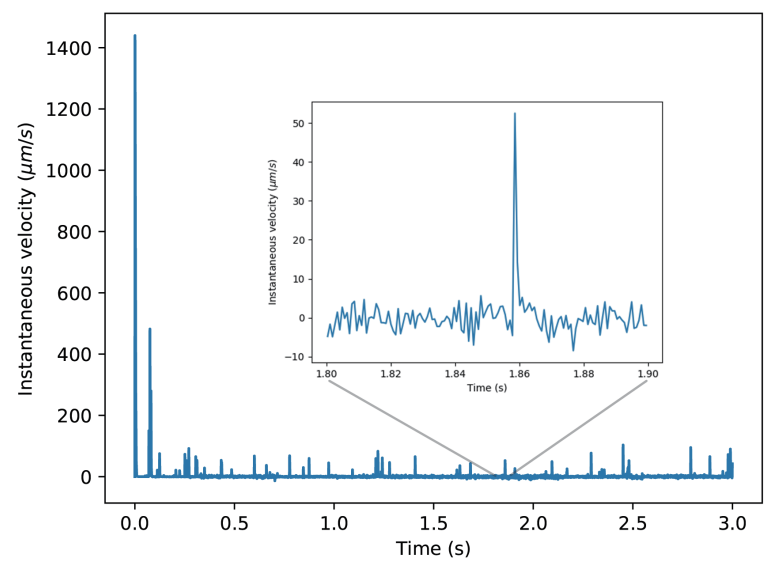

(b) $\gamma=400 s^{-1}$

Figure 4: The instantaneous velocity as a function of time at the shear rates of (a) $100 s^{-1}$ and (b) $400 s^{-1}$. The magnified drawings represent the instantaneous velocity within a 1-second period.

At a given shear rate, the rolling velocity increases as the flexural stiffness of the microvilli increases or as the flexibility of microvilli decreases, because the microvilli with a larger flexural stiffness have a smaller bending deformation and are likely to be oriented more vertically on the cell surface. Thus, the contact area and number of bonds between the cell and the substrate all become smaller, making the cell roll faster, as compared to cells with more flexible microvilli. The results of the number of bonds and the contact areas are viewed in Fig. 5 (b) and (c), where the number of bonds and the contact areas dramatically decrease as the flexural stiffness increases.

Next, the results of the deformation index as a function of the shear rate are given in Fig. 6 . The figure shows that at each of the given levels of flexural stiffness, the deformation index increases as the shear rate increases, an outcome which is consistent with the previous findings by Jadhav et al. (8). The deformation index increases by $10-11 \%$ when the shear rate increases from $\gamma=100 s^{-1}$ to $\gamma=400 s^{-1}$. However, at each of the given shear rates, when the flexural stiffness is largely varied from $K=0.1 K_{\text {exp }}$ to $K=10 K_{\text {exp }}$, the deformation index has only $1-3 \%$ variation, illustrating that the cell bulk deformation is more sensitive to the shear rate than the flexural stiffness. In other words, the bulk cell deformation strongly depends on the shear rate and less significantly on the flexural stiffness.

For the purpose of visualization, the animations of leukocyte rolling at shear rates of $\gamma=100 s^{-1}$ and $300 s^{-1}$ are presented in movie S1 and movie S2 in the Supporting Material.

Subsequently, Fig. 7 illustrates the instantaneous velocity of leukocyte as a function of time at three different levels of the flexural stiffness of microvilli at the shear rate of $\gamma=300 \mathrm{~s}^{-1}$. As the flexural stiffness increases, the time pauses become shorter, peaks are higher, and the rolling velocity in the case of $K=0.1 K_{\exp }$ experiences more frequent fluctuations between peaks, due to more occurrences of bonding and de-bonding, as compared to the velocities in the cases of $K=K_{\exp }$ and $K=10 K_{\text {exp }}$.

\section{Effects of the Flexural Stiffness of Microvilli on Bond Forces}

A total adhesive bond force is based on the global coordinates of X, Y, and Z and defined by

$$
\boldsymbol{F}^{\text {bond }}=\left\langle\sum_{i} \boldsymbol{F}_{b}^{i}\right\rangle
$$

where $i$ denotes the $i$ th bond.

In order to distinguish bending force from extensional force, a bond force can be decomposed into two components in a local coordinate system associated with a microvillus: one is parallel to the direction of the angular

Biophysical Journal 00(00) 1-22 


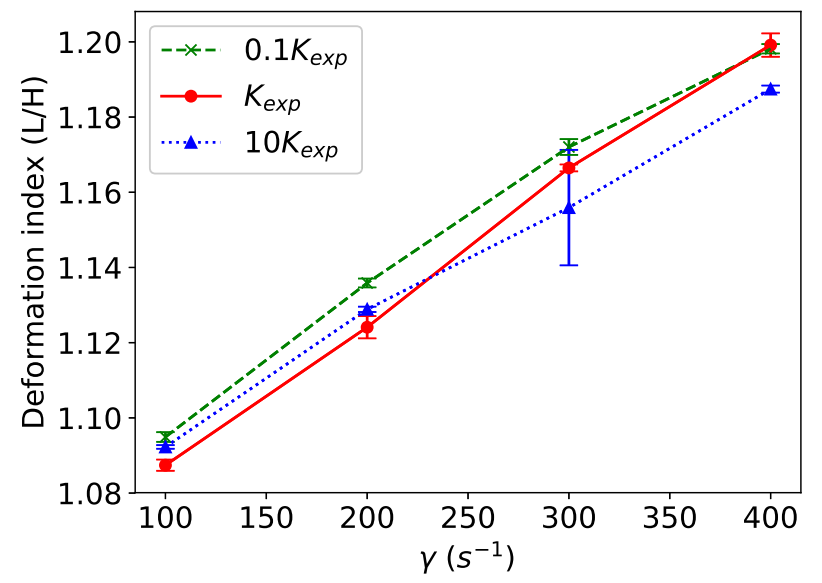

Figure 6: The simulation results of the deformation index at three different levels of the flexural stiffness of microvilli at the shear rates from $100 s^{-1}$ to $400 s^{-1}$.

The total adhesive bond force $\boldsymbol{F}^{\text {bond }}$, total local coordinate-based bond force $F_{L}^{\text {bond }}$, its parallel component $F_{L \|}^{b o n d}$, and perpendicular component $F_{L \perp}^{b o n d}$ are computed at three different levels of flexural stiffness, and the results as a function of shear rate are displayed in Fig. 8 (a), (b), (c), and (d), respectively. At each of the given levels of the flexural stiffness, the results of all the bond forces increase monotonically as the shear rate increases. This occurs because the adhesive bond force should be balanced by the hydrodynamic shear force, which drives the cell to move and induces a responding adhesive bond force. The larger the shear rate, the larger the responding adhesive bond force. Significantly, the bond force strongly depends on shear rates. In this study, the Reynolds number is $R e \in\left[1.172 \times 10^{-3}, 4.688 \times 10^{-3}\right]$, and therefore the inertial effect can be neglected.

The study confirms that the bond force is influenced not only by the shear rate but also by the flexural stiffness. At the given lowest shear rate of $\gamma=100 \mathrm{~s}^{-1}$, the result of the bond force increases monotonically as the flexural stiffness decreases and arrives at a maximum at the lowest level of the flexural stiffness of $K=0.1 K_{\exp }$. This result is due to the largest bending deformation of microvilli (see the next section), which may result in a larger contact area and a larger bonding probability. At the given highest level of the shear rate of $\gamma=400 s^{-1}$, the bond force increases first as the flexural stiffness decreases from $K=10 K_{\exp }$ to $K_{\exp }$ and then decreases as the flexural stiffness continuously decreases to the level of $K=0.1 K_{\text {exp }}$. The maximum bond force occurs at an intermediate level of the flexural stiffness. A similar behavior is observed at $\gamma=200 s^{-1}$ and $300 s^{-1}$. It is observed that across the entire shear rate range, the amplitude of the increase in $\boldsymbol{F}^{\text {bond }}$ and $F_{L}^{b o n d}$ is largest for the case of the intermediate flexural stiffness of $K=K_{\text {exp }}$. If the flexural stiffness is very small $\left(K=0.1 K_{\text {exp }}\right)$ or the microvilli are too flexible, the bending deformation is saturated even at the small shear rate and lends to a negligible difference when compared to the large shear rates. Similarly, if the flexural stiffness is very large or the microvilli are too stiff, the bending deformation lends to a negligible difference across all the shear rates. Importantly, the maximum amplitude of the increase of the bond force occurs at the flexural stiffness of the experimental value of $K=K_{\exp }$. It is inferred that an interplay of the roles between the flexural stiffness and flow shear rate determines the final total bond force.

As shown in Fig. 8 (d), the perpendicular component forces are profoundly affected by the flexural stiffness at each of the given shear rates. For example, at the lowest level of the shear rate of $\gamma=100 s^{-1}$, the perpendicular component increases from $80 p N$ to $185 p N$, as the flexural stiffness continuously decreases from $K=10 K_{\text {exp }}$ to $K=0.1 K_{\text {exp }}$, while the parallel component just slightly changes from $122 p N$ to $129 p N$, as shown in Fig. 8 (c). The net force change due to the flexural stiffness is 15 times larger in the perpendicular component than in the parallel component. It is concluded that the change in the total bond force primarily results from the change in the perpendicular component. This outcome can also be seen from the case of the shear rate of $400 \mathrm{~s}^{-1}$. The perpendicular component increases from $331 p N$ to $518 p N$, while the parallel component changes only from $433 p N$ to $359 p N$. Again, the increase in the perpendicular component is much larger than the change in the parallel component due to the bending of microvilli. In fact, the bending angle significantly increases as the flexural stiffness decreases or as the flexibility increases, as discussed in the next section.

Biophysical Journal 00(00) 1-22 


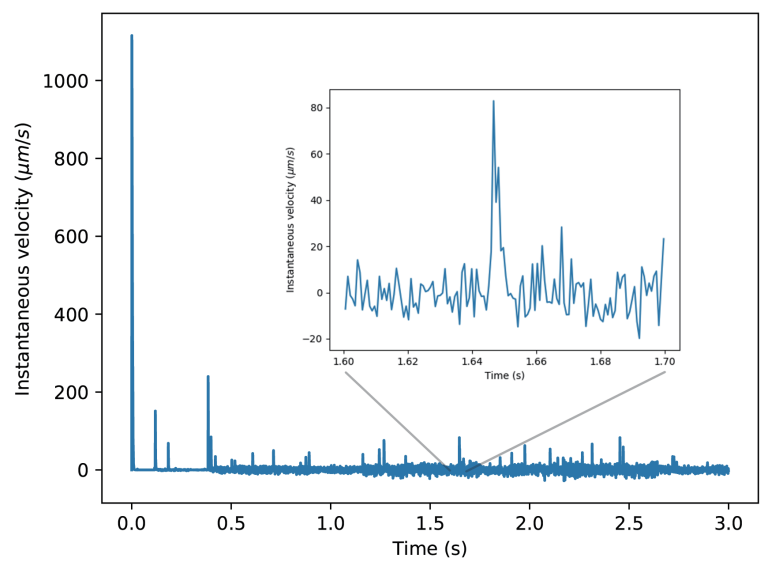

(a) $0.1 K_{\exp }$

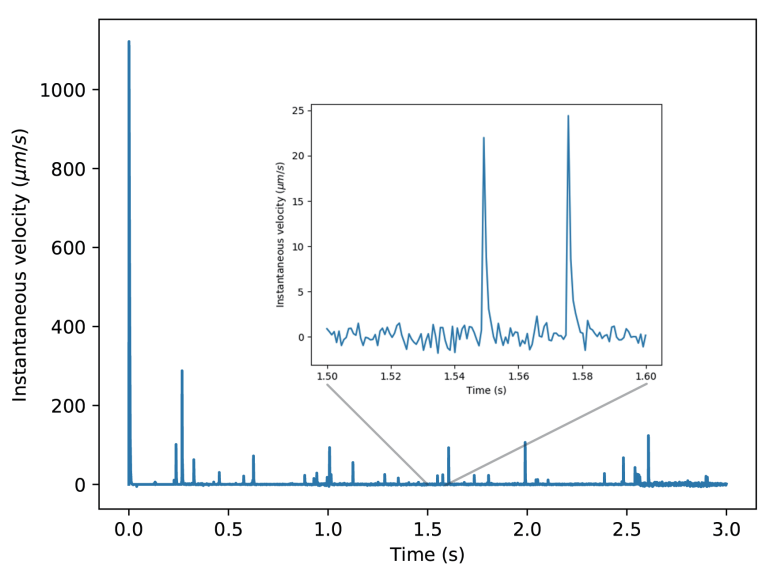

(b) $K_{\exp }$

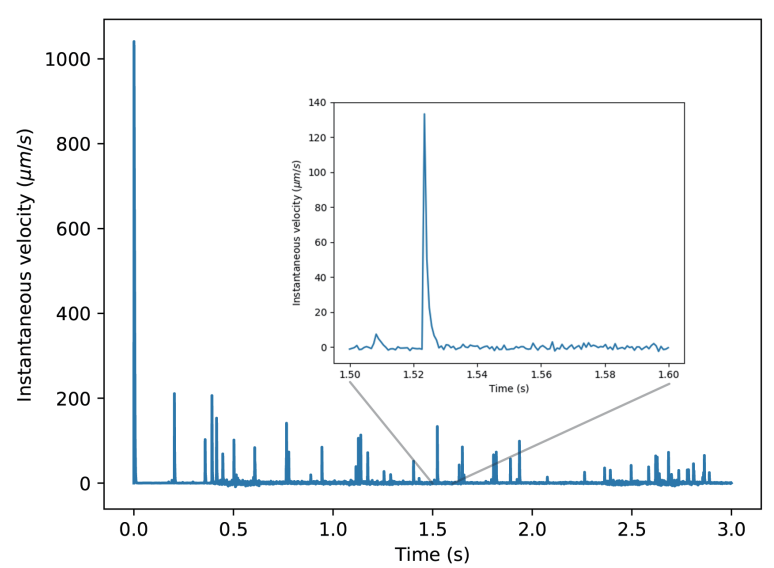

(c) $10 K_{\text {exp }}$

Figure 7: TThe instantaneous velocity at microvillus flexural stiffness of (a) $0.1 K_{\text {exp }}$, (b) $K_{\text {exp }}$, and (c) $10 K_{\text {exp }}$ at the shear rate of $\gamma=300 \mathrm{~s}^{-1}$. The magnified drawing presents the instantaneous velocity in 0.1 -second period.

\section{Bending Deformation of Microvilli}

In order to examine the influence of the flexural stiffness of microvilli on their bending deformation, an angular distribution function $f(\theta)$ of the bounded microvilli is defined by

$$
f(\theta)=\frac{1}{\pi n} \sum_{i} \delta\left(\theta-\theta_{i}\right)
$$

where $n$ is the total number of the bonded microvilli, and $\theta_{i}$ is the bending angle of the $i$ th bonded microvillus. The angular distribution function is utilized to describe the probability of finding a microvillus at an angle of $\theta$ per unit angle.

The results of an ensemble average of the angular distribution function $\langle f(\theta)\rangle$, as a function of the bending angle at three different levels of the flexural stiffness, are displayed and compared in Fig. 9, at each of the four different shear rates, where the green dashed, red solid, and blue dash-dot lines represent the data sets corresponding to $K=0.1 K_{\text {exp }}, K_{\text {exp }}$, and $10 K_{\text {exp }}$. It is shown that the flexural stiffness has a great impact on the bending deformation of microvilli. For example, for the case of the shear rate of $\gamma=100 \mathrm{~s}^{-1}$, three curves of the angular distribution function are compared in Fig. 9 (a). One sharp peak appears on the curve of the largest flexural stiffness of $K=10 K_{\text {exp }}$. This peak value is as high as 0.95 and is located near the angle of zero, evidencing that most 


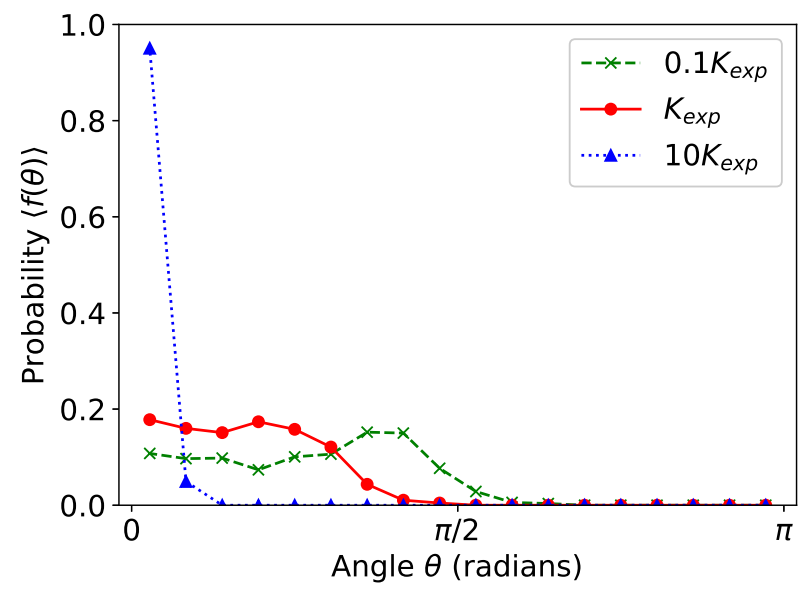

(a) $\gamma=100 s^{-1}$

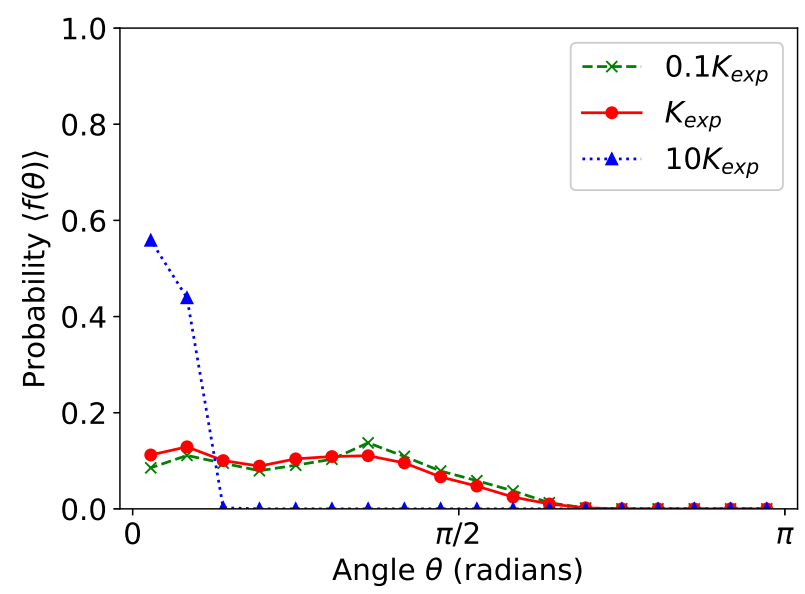

(c) $\gamma=300 s^{-1}$

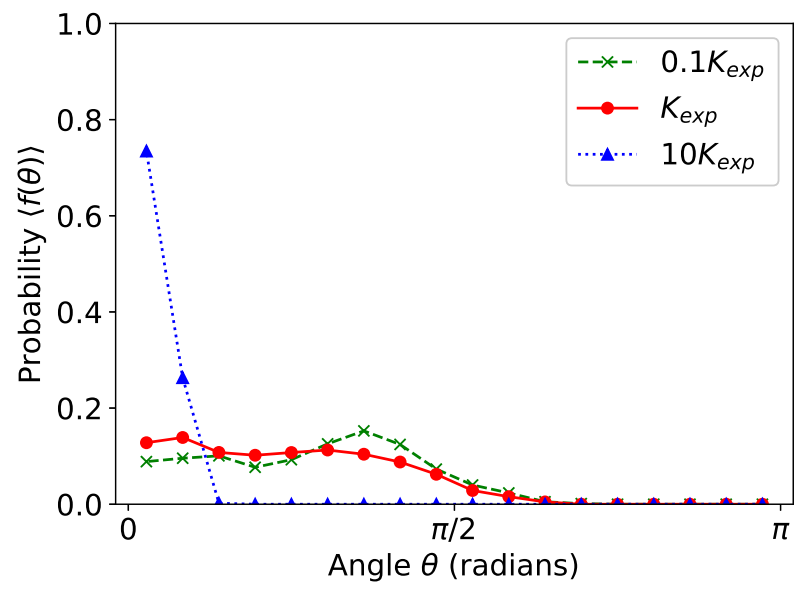

(b) $\gamma=200 s^{-1}$

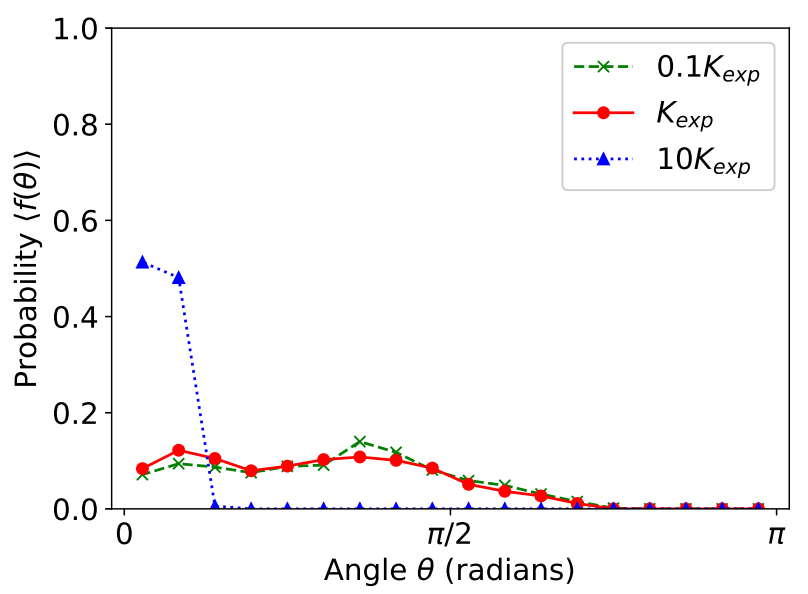

(d) $\gamma=400 s^{-1}$

Figure 9: The simulation results of the angular distribution of the microvilli at three different levels of the microvillus flexural stiffness at shear rates of (a) $100 s^{-1}$, (b) $200 s^{-1}$, (c) $300 s^{-1}$, and (d) $400 s^{-1}$.

and blue dotted lines represent the duration involved in bonded and non-bonded states, respectively. Each figure also includes both the side and bottom views of the leukocyte. For the bottom view, the red marker specifies the particularly selected single microvillus (e.g., microvilli No. 1, 2, 3, and 4), and the black points denote the other microvilli. Microvillus No. 1 is initially located near the center of the contact area, while No. 2 is initially located near the right edge of the contact area. The same shear rate $\gamma=400 s^{-1}$ is applied on microvilli No. 1 and 2 . Microvilli No. 3 and 4 are similar to No. 1 and 2, except that the smaller shear rate $\gamma=100 \mathrm{~s}^{-1}$ is used.

The variation of the bending angle of microvillus No. 1 is shown in Fig. 10 (a) as the microvillus moves from the center to the left edge of the contact area during the rolling process. In the initial period $(t<0.12 s)$, there is non-bonding involved (blue dotted line). The microvillus stands vertically on the cell surface with a very small bending angle and then is quickly bent with a large angle and flattened with the surface. Soon after that, a receptorligand bond is developed and formed at $t=0.12 \mathrm{~s}$. As the leukocyte continues to roll, the angle of the microvillus first decreases and then increases. Finally, the bond is broken at $t=2.17 \mathrm{~s}$ and the microvillus becomes vertical again with a small bending angle that occurs when the microvillus moves to the left edge of the contact area. This non-bonding, bonding, and de-bonding process corresponds to the stop-and-go motion. The bending behavior of microvillus No. 2, moving from the right edge to the center of the contact area during the rolling process, is shown in Fig. 10 (b). A bond is formed on the vertical microvillus at $t=1.31 \mathrm{~s}$. Unlike No. 1 , the bending angle increases 
three different levels, while the extensional stiffness is fixed at a constant, allowing one's attention to be focused on the effects of flexural stiffness only.

At each given level of the flexural stiffness, the flow shear rate is varied at four different levels, and the results of the bond force, number of bonds, contact area, bending angular distribution, and cell deformation index are computed. To address the bending, the bond force is decomposed into two directions, parallel and perpendicular to the direction of cell surface in an associated local coordinate, so that the perpendicular force component is separated from the parallel force component, and the role of the perpendicular force component on bending is extracted and analyzed.

Several interesting behaviors are revealed from simulations:

1. At each of the given levels of the flexural stiffness, the rolling velocity, total bond force, number of bonds, bending angles, and contact area increase as the shear rate increases.

2. At a given shear rate, the flexural stiffness also significantly affects the bond force, bending deformation, number of bonds, and contact area. The angular distribution function is employed to statistically measure the bending deformation. The position of the peak of the curve of the angular distribution function of the microvilli shifts to the side of $\theta=\pi / 2$, the value of the peak reduces, and the peak becomes wider and lower, as the stiffness reduces, illustrating that the microvilli become more flattened with the surface and have a larger probability to be bonded with the substrates.

3. The interplay of the roles between the shear rate and flexural stiffness of microvilli determines the final bond force.

4. It is demonstrated that the bending deformation and perpendicular force component strongly depend on the flexural stiffness, while the bulk cell deformation mainly depends on shear rates and is not sensitive to the flexural stiffness of microvilli.

The above information may improve the efficacy of diagnoses and treatments, especially within the realm of technological advances, such as the design of microfluidic chips.

\section{SUPPLEMENTARY MATERIAL}

Two movies are available at http://www.biophysj.org.

\section{ACKNOWLEDGMENTS}

Wu and Qi thank Dr. M. Di Pierro and Yi-Chin Huang for proofreading and writing advise. Wu also acknowledges the Gwen Frostic Doctoral Fellowship for partially supporting this work and Qi acknowledges the award of Faculty Creativity Initiative of College of Engineering and Applied Sciences at the Western Michigan University. 


\section{References}

[1] Kochenderfer, J. N., W. H. Wilson, J. E. Janik, M. E. Dudley, M. Stetler-Stevenson, S. A. Feldman, I. Maric, M. Raffeld, D.-A. N. Nathan, B. J. Lanier, R. A. Morgan, and S. A. Rosenberg. 2010. Eradication of b-lineage cells and regression of lymphoma in a patient treated with autologous t cells genetically engineered to recognize cd19. Blood. 116:4099-4102.

[2] Jackson, H. J., S. Rafiq, and R. J. Brentjens. 2016. Driving CAR T-cells forward. Nature Reviews Clinical Oncology. 13:370-383.

[3] Hammer, D., and S. Apte. 1992. Simulation of cell rolling and adhesion on surfaces in shear flow: general results and analysis of selectin-mediated neutrophil adhesion. Biophys. J. 63:35 - 57.

[4] Chang, K.-C., D. F. J. Tees, and D. A. Hammer. 2000. The state diagram for cell adhesion under flow: Leukocyte rolling and firm adhesion. Proceedings of the National Academy of Sciences. 97:11262-11267.

[5] Bhatia, S. K., M. R. King, and D. A. Hammer. 2003. The state diagram for cell adhesion mediated by two receptors. Biophysical Journal. 84:2671 - 2690.

[6] Khismatullin, D. B., and G. A. Truskey. 2004. A 3d numerical study of the effect of channel height on leukocyte deformation and adhesion in parallel-plate flow chambers. Microvascular Research. 68:188 - 202.

[7] Khismatullin, D. B., and G. A. Truskey. 2005. Three-dimensional numerical simulation of receptor-mediated leukocyte adhesion to surfaces: Effects of cell deformability and viscoelasticity. Physics of Fluids. 17:031505.

[8] Jadhav, S., C. D. Eggleton, and K. Konstantopoulos. 2005. A 3-d computational model predicts that cell deformation affects selectin-mediated leukocyte rolling. Biophysical Journal. 88:96 - 104.

[9] Park, E. Y., M. J. Smith, E. S. Stropp, K. R. Snapp, J. A. DiVietro, W. F. Walker, D. W. Schmidtke, S. L. Diamond, and M. B. Lawrence. 2002. Comparison of psgl-1 microbead and neutrophil rolling: Microvillus elongation stabilizes p-selectin bond clusters. Biophysical Journal. 82:1835 - 1847.

[10] Yago, T., A. Leppänen, H. Qiu, W. D. Marcus, M. U. Nollert, C. Zhu, R. D. Cummings, and R. P. McEver. 2002. Distinct molecular and cellular contributions to stabilizing selectin-mediated rolling under flow. The Journal of Cell Biology. 158:787-799.

[11] Pappu, V., and P. Bagchi. 2008. 3d computational modeling and simulation of leukocyte rolling adhesion and deformation. Computers in Biology and Medicine. 38:738 - 753.

[12] Pappu, V., S. K. Doddi, and P. Bagchi. 2008. A computational study of leukocyte adhesion and its effect on flow pattern in microvessels. Journal of Theoretical Biology. 254:483-498.

[13] Fedosov, D., B. Caswell, and G. Karniadakis. 2011. Wall shear stress-based model for adhesive dynamics of red blood cells in malaria. Biophysical Journal. 100:2084 - 2093.

[14] Luo, Z. Y., S. Q. Wang, L. He, T. J. Lu, F. Xu, and B. F. Bai. 2013. Front tracking simulation of cell detachment dynamic mechanism in microfluidics. Chemical Engineering Science. 97:394- 405.

[15] Luo, Z. Y., and B. F. Bai. 2016. State diagram for adhesion dynamics of deformable capsules under shear flow. Soft matter. 12:6918-6925.

[16] Xiao, L. L., Y. Liu, S. Chen, and B. M. Fu. 2017. Effects of flowing rbcs on adhesion of a circulating tumor cell in microvessels. Biomechanics and Modeling in Mechanobiology. 16:597-610.

[17] Caputo, K. E., and D. A. Hammer. 2005. Effect of microvillus deformability on leukocyte adhesion explored using adhesive dynamics simulations. Biophysical Journal. 89:187 - 200.

[18] Shao, J.-Y., H. P. Ting-Beall, and R. M. Hochmuth. 1998. Static and dynamic lengths of neutrophil microvilli. Proceedings of the National Academy of Sciences. 95:6797-6802.

[19] Pawar, P., S. Jadhav, C. D. Eggleton, and K. Konstantopoulos. 2008. Roles of cell and microvillus deformation and receptor-ligand binding kinetics in cell rolling. American Journal of Physiology - Heart and Circulatory Physiology. 295:H1439-H1450.

[20] POSPIESZALSKA, M. K., A. ZARBOCK, J. E. PICKARD, and K. LEY. 2009. Event-tracking model of adhesion identifies load-bearing bonds in rolling leukocytes. Microcirculation. 16.

[21] Pospieszalska, M. K., and K. Ley. 2009. Dynamics of microvillus extension and tether formation in rolling leukocytes. Cellular and Molecular Bioengineering. 2:207.

[22] Shao, J.-Y. 2009. Chapter 2 biomechanics of leukocyte and endothelial cell surface. In Current Topics in Membranes, Current Topics in Membranes, volume 64. Academic Press. $25-45$.

[23] Munn, L., R. Melder, and R. Jain. 1996. Role of erythrocytes in leukocyte-endothelial interactions: mathematical model and experimental validation. Biophysical Journal. 71:466 - 478.

[24] Yao, D.-K., and J.-Y. Shao. 2007. Flexibility of single microvilli on live neutrophils and lymphocytes. Phys. Rev. E. 76:021907.

[25] Yao, D.-K., and J.-Y. Shao. 2008. A novel technique of quantifying flexural stiffness of rod-like structures. Cellular and Molecular Bioengineering. 1:75-83.

[26] Simon, S. I., T. Nyunt, K. Florine-Casteel, K. Ritchie, H. P. Ting-Beall, E. Evans, and D. Needham. 2007. Dynamics of neutrophil membrane compliance and microstructure probed with a micropipet-based piconewton force transducer. Ann. Biomed. Eng. 35:595-604.

[27] Wu, T.-H., and D. Qi. 2018. Role of flexural stiffness of leukocyte microvilli in adhesion dynamics. Phys. Rev. Fluids.

Biophysical Journal 00(00) 1-22 
3:031101.

[28] Qi, D. 1999. Lattice boltzmann simulations of particles in non-zero reynolds number flows. J. Fluid Mech. 385:41.

[29] Lallemand, P., and L.-S. Luo. 2000. Theory of the lattice boltzmann method: Dispersion, dissipation, isotropy, galilean invariance, and stability. Phys. Rev. E. 61:6546-6562.

[30] Succi, S. 2001. The lattice Boltzmann equation: for fluid dynamics and beyond. Oxford university press.

[31] QI, D., and L.-S. LUO. 2003. Rotational and orientational behaviour of three-dimensional spheroidal particles in couette flows. Journal of Fluid Mechanics. 477:201;V213.

[32] Qi, D. 2006. Direct simulations of flexible cylindrical fiber suspensions in finite reynolds number flows. J. Chem. Phys. 125:114901-10.

[33] Qi, D., Y. Liu, W. Shyy, and H. Aono. 2010. Simulations of dynamics of plunge and pitch of a three-dimensional flexible wing in a low reynolds number flow. Physics of Fluids. 22:091901.

[34] Aidun, C. K., and J. R. Clausen. 2010. Lattice-boltzmann method for complex flows. Annual review of fluid mechanics. 42:439-472.

[35] Guo, Z., and C. Shu. 2013. Lattice Boltzmann method and its applications in engineering, volume 3. World Scientific.

[36] Qi, D., G. He, and Y. Liu. 2014. Lattice boltzmann simulations of a pitch-up and pitch-down maneuver of a chord-wise flexible wing in a free stream flow. Phys. Fluids. 26:021902.

[37] Qi, D., and R. Gordnier. 2015. Effects of deformation on lift and power efficiency in a hovering motion of a chord-wise flexible wing. Journal of Fluids and Structures. 54:142-170.

[38] Fedosov, D. A., B. Caswell, and G. E. Karniadakis. 2010. A multiscale red blood cell model with accurate mechanics, rheology, and dynamics. Biophysical Journal. 98:2215 - 2225.

[39] Fedosov, D. A. 2010. Multiscale Modeling of Blood Flow and Soft Matter. Brown University. :292.

[40] Fedosov, D. A., J. Fornleitner, and G. Gompper. 2012. Margination of white blood cells in microcapillary flow. Phys. Rev. Lett. 108:028104.

[41] Fedosov, D. A., and G. Gompper. 2014. White blood cell margination in microcirculation. Soft Matter. 10:2961-2970.

[42] Wu, T.-H., R.-S. Guo, G.-W. He, Y.-M. Liu, and D. Qi. 2014. Simulation of swimming of a flexible filament using the generalized lattice-spring lattice-boltzmann method. J. Theor. Biol. 349:1 - 11.

[43] Peskin, C. S. 2002. The immersed boundary method. Acta Numerica. 11:479-517.

[44] Wu, T.-H., and D. Qi. 2017. Lattice-boltzmann lattice-spring simulations of influence of deformable blockages on blood fluids in an elastic vessel. Computers \& Fluids. 155:103 - 111.

[45] Luo, Y., T.-H. Wu, and D. Qi. 2017. Lattice-boltzmann lattice-spring simulations of flexibility and inertial effects on deformation and cruising reversal of self-propelled flexible swimming bodies. Computers \& Fluids. 155:89 - 102.

[46] Tang, Y., T.-H. Wu, G.-W. He, and D. Qi. 2018. Multi-flexible fiber flows: A direct-forcing immersed boundary lattice-boltzmann lattice-spring approach. International Journal of Multiphase Flow. 99:408 - 422.

[47] Sun, C., and L. L. Munn. 2005. Particulate nature of blood determines macroscopic rheology: A 2-d lattice boltzmann analysis. Biophys. J. 88:1635 - 1645 .

[48] Sun, C., and L. L. Munn. 2006. Influence of erythrocyte aggregation on leukocyte margination in postcapillary expansions: A lattice boltzmann analysis. Physica A: Statistical Mechanics and its Applications. 362:191 - 196 . Discrete Simulation of Fluid Dynamics.

[49] Dupin, M. M., I. Halliday, C. M. Care, L. Alboul, and L. L. Munn. 2007. Modeling the flow of dense suspensions of deformable particles in three dimensions. Phys. Rev. E. 75:066707.

[50] Dupin, M., I. Halliday, C. Care, and L. Munn. 2008. Lattice boltzmann modelling of blood cell dynamics. International Journal of Computational Fluid Dynamics. 22:481-492.

[51] Munn, L. L., and M. M. Dupin. 2008. Blood cell interactions and segregation in flow. Ann. Biomed. Eng. 36:534-544.

[52] D'Humières, D., I. Ginzburg, M. Krafczyk, P. Lallemand, and L.-S. Luo. 2002. Multiple-relaxation-time lattice Boltzmann models in three dimensions. Philosophical transactions. Series A, Mathematical, physical, and engineering sciences. 360:437-451.

[53] Pan, C., L.-S. Luo, and C. T. Miller. 2006. An evaluation of lattice boltzmann schemes for porous medium flow simulation. Computers \& Fluids. 35:898 - 909. Proceedings of the First International Conference for Mesoscopic Methods in Engineering and Science.

[54] Guo, Z., C. Zheng, and B. Shi. 2002. Discrete lattice effects on the forcing term in the lattice boltzmann method. Phys. Rev. E. 65:046308.

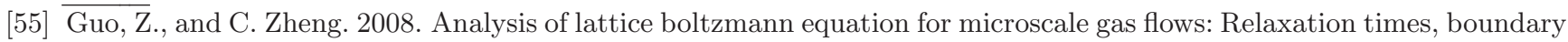
conditions and the knudsen layer. International Journal of Computational Fluid Dynamics. 22:465-473.

[56] Persson, P.-O., and G. Strang. 2004. A simple mesh generator in matlab. SIAM Review. 46:329-345.

[57] Dembo, M., D. C. Torney, K. Saxman, and D. Hammer. 1988. The reaction-limited kinetics of membrane-to-surface adhesion and detachment. Proceedings of the Royal Society of London B: Biological Sciences. 234:55-83.

[58] Skalak, R., and S. Chien. 1987. Handbook of bioengineering. McGraw-Hill Companies.

[59] Tandon, P., and S. L. Diamond. 1998. Kinetics of £]2-integrin and l-selectin bonding during neutrophil aggregation in shear flow. Biophysical Journal. 75:3163 - 3178. 
